\title{
Health uncertainty among healthcare workers during the COVID-19 pandemic
}

\author{
Daniel L. Hall*1,2,3 , Christina M. Luberto ${ }^{1,2,3}$, Alexandros Markowitz ${ }^{2}$, Helen Mizrach ${ }^{2}$, Nevita George ${ }^{4}$, Giselle K. \\ Perez $^{1,2,3}$, Nicole R. DeTore ${ }^{1}$, Gregory L. Fricchione ${ }^{1,3}$, Daphne Holt ${ }^{1}$, Louisa Sylvia ${ }^{1,4}$, Elyse R. Park ${ }^{1,2,3}$ \\ ${ }^{1}$ Harvard Medical School, Boston, MA, United States \\ ${ }^{2}$ Health Promotion and Resiliency Intervention Research, Mongan Institute, Massachusetts General Hospital, Boston, MA, \\ United States \\ ${ }^{3}$ Benson-Henry Institute for Mind Body Medicine, Massachusetts General Hospital, Boston, MA, United States \\ ${ }^{4}$ Dauten Family Center for Bipolar Treatment Innovation, Massachusetts General Hospital, Boston, MA, United States
}

Received: March 12, 2021

DOI: $10.5430 /$ jha.v10n2p45
Accepted: April 5, 2021

URL: https://doi.org/10.5430/jha.v10n2p45

\begin{abstract}
Objective: Health uncertainty among healthcare workers has yet to be examined as a contributor to the psychological toll of the COVID-19 pandemic. We aimed to (1) characterize health uncertainty levels among healthcare workers in a large, U.S. hospital system during the COVID-19 pandemic and (2) examine associations between health uncertainty and psychological outcomes. Methods: From March to June 2020, healthcare workers in a large, urban U.S. healthcare system were invited to complete an online questionnaire. Self-report measures assessed sociodemographic characteristics and job roles, health uncertainty, and emotional wellbeing variables (anxiety, depression, loneliness, self-compassion, and coping confidence). Health uncertainty (categorical and continuous scores) was compared across each variable using correlations and ANOVAs.

Results: Healthcare workers $(\mathrm{N}=440)$ were on average 44.5 years of age, $88.9 \%$ female, and $84.5 \%$ non-Hispanic white. Over half $(52 \%)$ endorsed experiencing health uncertainty "sometimes" to "all the time". While unrelated to sociodemographic characteristics $(p s>.05)$, health uncertainty was highest among pharmacists and technicians, with levels significantly higher than other roles including physicians $(p<.05)$ and mental health and spiritual counselors $(p<.05)$. Higher health uncertainty was associated with higher anxiety $(p<.001)$, depression $(p<.001)$, and loneliness $(p<.001)$, higher self-compassion $(p=.02)$, and lower coping confidence $(p<.001)$.

Conclusions: Health uncertainty during the COVID-19 pandemic is common among healthcare workers, with higher levels related to poorer emotional wellbeing and less confidence in their coping abilities. Further research is needed to understand the relationships between healthcare workers' health uncertainties and associated factors (i.e., job roles) and to identify whether health uncertainty may be a modifiable target for future interventions.
\end{abstract}

Key Words: COVID-19, Health uncertainty, Coping, Healthcare workers, Emotional wellbeing

\section{INTRODUCTION}

The COVID-19 pandemic has amplified uncertainty in nearly every facet of society, impacting the daily lives of individuals around the globe. As a result, many have lost their sense of safety and security, which have been replaced with anxiety, doubt, and lack of control. There remain many unknowns sur-

\footnotetext{
*Correspondence: Daniel L. Hall; Email: hall@mgh.harvard.edu; Address: Massachusetts General Hospital, 100 Cambridge Street, Suite 1600, Boston, MA, 02114, United States.
} 
rounding the COVID-19 virus itself, as new strains emerge, and clinical and public health guidelines continue to change surrounding its etiology, management, treatment, and prevention. Healthcare workers in hospitals treating COVID-19 patients are a subpopulation who have no choice but to face these conditions head-on. Among an international sample of frontline clinicians, half reported burnout. ${ }^{[1]}$ Furthermore, record numbers requested Americans with Disabilities Act exemptions, which is reflective of the burden placed upon them during this time. ${ }^{[2]}$ It is no surprise that healthcare workers are facing challenges to their emotional wellbeing during the pandemic, including elevated levels of distress, anxiety, insomnia, and depressive symptoms. ${ }^{[3,4]}$ Other largescale epidemics have also been associated with significant increases in mental health disorders among care providers-in the immediate aftermath and over long periods of time. ${ }^{[5]}$

Uncertainty is a key factor in the distress faced during COVID-19. Shechter et al. ${ }^{[6]}$ found that in the clinical environment, uncertainty about colleagues' COVID-19 status was commonly identified as a source of stress. The national unavailability of COVID-19 testing capabilities, limited PPE, and lack of national treatment guidelines for patients also contributed to increased uncertainty. ${ }^{[6]}$ The degree to which healthcare workers experience health uncertainty (i.e., uncertainty about one's own physical health), however, has yet to be examined as a contributor to the psychological toll of the pandemic. Health uncertainty is known to contribute to elevated psychological distress in other populations, such as advanced cancer patients and parents of children with chronic health conditions. ${ }^{[7-9]}$ Although all healthcare workers are susceptible to the psychological toll of health uncertainty, the psychological burden of uncertainty may vary by the clinical, research, and administrative roles that comprise a healthcare system. ${ }^{[6,10]}$ Indeed, unique COVID-19 related concerns vary across different groups of healthcare workers but studies have not directly compared experiences of health uncertainty between clinical roles. ${ }^{[11-13]}$

According to empirically supported cognitive-behavioral models of health anxiety, situations that involve novelty, uncontrollability, or unpredictability are known to trigger uncertainty, which the brain attempts to resolve by making assumptions. ${ }^{[14]}$ When conditions are stressful, these assumptions tend to characterize the future as dangerous and overestimate the probability of disaster, leading to increased anxiety and other distressing mood states. ${ }^{[15,16]}$ For healthcare workers, the prolonged uncertainties brought on by the COVID-19 pandemic may create conditions for chronic stress responses that make healthcare workers vulnerable to poorer long-term emotional and physical wellbeing. ${ }^{[17]}$ Given the unique and sustained uncertainties faced by health- care workers during the COVID-19 pandemic, they may be particularly vulnerable to poorer emotional wellbeing and difficulties coping, although to date these associations remain unexamined.

The virus is unrelenting in the U.S., and the nation has entered subsequent waves that have become more severe than the first. Therefore, it is critical to gain a better understanding of health uncertainty among healthcare workers, in order to determine how and for whom to tailor proactive and preventative mental health services. In this report, we aimed to (1) characterize levels of health uncertainty among healthcare workers in a large, U.S. hospital system during the COVID-19 pandemic, and (2) examine potential sociodemographic, job-related, and emotional wellbeing correlates of higher health uncertainty levels within this cohort. Based on cognitive-behavioral models tested by our group ${ }^{[18]}$ and others, ${ }^{[16]}$ we hypothesized that higher health uncertainty would be associated with poorer emotional wellbeing.

\section{METHODS}

This study was conducted at the Mass General Brigham healthcare system in Boston, Massachusetts. The study protocol was approved by a COVID internal board review and the Mass General Brigham Institutional Review Board.

\subsection{Recruitment}

English-speaking healthcare workers over 18 years of age in the Mass General Brigham healthcare system during COVID19 were eligible, including physicians, nurses, medical assistants, advanced practitioners, therapists (physical therapists, occupational therapists, respiratory therapists, speech language pathologists), pharmacists, mental health clinicians, spiritual counselors, technicians, and research and administrative staff. Recruitment occurred from 3/23/20-7/30/20 through hospital emails and departmental announcements publicizing an asynchronous online resiliency intervention as part of a separate study. Interested clinicians registered online and completed a questionnaire via REDCap upon study entry.

\subsection{Measures}

Sociodemographic and Job Role. Age, sex, race/ethnicity, and job role were assessed.

Health Uncertainty. Health uncertainty was measured by adapting a validated single item measure of fear of cancer recurrence ${ }^{[19]}$ to assess participants' uncertainty about their health. The item "Please indicate your uncertainty about your health" includes responses on a 5-point scale (I have no uncertainty about my health $=0$, I have a little uncertainty about my health, with occasional thoughts but they don't 
really bother me $=1$, I sometimes have uncertainty about my health, but I can usually manage it $=2$, I have a lot of uncertainty about my health, and it can really preoccupy my thoughts $=3$, I am uncertain about my health all the time and I struggle with this $=4)$; range $=0-4$.

Anxiety and Depressive Symptoms. The Patient Health Questionnaire (PHQ-4) ${ }^{[20]}$ assesses anxiety ("feeling nervous, anxious or on edge" and "not being able to stop or control worrying"; GAD-2 subscale) and depressive symptoms ("feeling down, depressed, or hopeless" and "little interest or pleasure in doing things"; PHQ-2 subscale) over the past two weeks on a 4-point scale (Not at All $=0$, Several Days $=1$, More than Half the Days $=2$, Nearly Every Day $=3$ ). Subscale scores for anxiety (range $=0-6)$ and depression (range $=0-6$ ) were created by summing the two items associated with each emotional state; range $=0-4$.

Loneliness. The UCLA Loneliness Scale (UCLA) ${ }^{[21]}$ measures subjective feelings of loneliness, social isolation, and disconnectedness from others. Two items ("I feel completely alone" and "I feel isolated from others") on a 4-point scale (Not at All =0, Several Days $=1$, More than Half the Days $=2$, Nearly Every Day $=3$ ) were summed to create a total score; range $=0-6$.

Self-Compassion. The Self-Compassion Scale (SCS) ${ }^{[22]}$ measures the ability to extend warmth and understanding to one's self under difficult circumstances. We used one item from the SCS ("When times are really difficult, I am tough on myself"). This item was rated on a 5-point Likert scale (Strongly Disagree $=1$, Disagree $=2$, Neutral $=3$, Agree $=4$, Strongly Agree $=5$ ) and was reverse-scored, such that higher scores reflect higher levels of self-compassion; range $=1-5$.

Coping Confidence. The Measure of Current Status (MOCSA) ${ }^{[23]}$ assesses one's ability to choose appropriate coping responses and restructure maladaptive thoughts. Two items ("I am confident about being able to choose the best coping response for hard situations" and "I can come up with emotionally balanced thoughts even during negative times") were rated on a 5 -point scale $(1=$ Cannot do this at all; $2=\mathrm{I}$ can do this just a little bit; $3=\mathrm{I}$ can do this a medium amount; 4 $=\mathrm{I}$ can do this pretty well; $5=\mathrm{I}$ can do this extremely well) and summed to create a total score; range $=2-10$.

\subsection{Statistical analyses}

All analyses were conducted using SPSS (v24). Distributions of variables were assessed for normality (e.g., skew, kurtosis). All variables were found to be normally distributed. Descriptive statistics included computation of means and standard deviations to characterize levels of health uncertainty on the continuous 0-4 scale. In addition, to provide an estimate of rates of elevated health uncertainty, health uncertainty scores were dichotomized ("A little" or less vs "Sometimes" or more) analyzed using frequencies and percentages. For Aim 2, correlations (Pearson's r; for continuous variables) and ANOVAs (for categorical variables) were computed to test associations of sociodemographic, job role, and psychosocial variables with the health uncertainty continuous score. Statistical significance was determined using an alpha of .05. A significant omnibus ANOVA of health uncertainty among job roles was probed with Tukey's HSD test to compare mean differences in health uncertainty, applying a Bonferroni correction to adjust for multiple comparisons.

Table 1. Characteristics of healthcare workers $(\mathrm{N}=440)$

\begin{tabular}{|c|c|}
\hline & $\mathbf{N}(\%) / \mathrm{M}(S D)$ \\
\hline Age (years) & $44.5(13.1)$ \\
\hline Gender (female) & $391(88.9 \%)$ \\
\hline \multicolumn{2}{|l|}{ Race $^{*}$} \\
\hline American Indian/Alaska Native & $7(1.6 \%)$ \\
\hline White & $372(84.5 \%)$ \\
\hline Black & $12(2.7 \%)$ \\
\hline Asian & $38(8.6 \%)$ \\
\hline Prefer Not to Answer & $20(4.5 \%)$ \\
\hline \multicolumn{2}{|l|}{ Ethnicity } \\
\hline Hispanic & $30(6.8 \%)$ \\
\hline Non-Hispanic & $399(90.7 \%)$ \\
\hline Prefer Not to Answer & $11(2.5 \%)$ \\
\hline Number of People in Household ${ }^{* *}$ & $2.8(1.5)$ \\
\hline \multicolumn{2}{|l|}{ Job Role } \\
\hline Mental Health and Spiritual Counselor & $23(5.2 \%)$ \\
\hline Nurse, Medical Assistant, Advanced Practitioner & $164(37.3 \%)$ \\
\hline Pharmacist & $7(1.6 \%)$ \\
\hline Physician & $78(17.7 \%)$ \\
\hline Research/Administrative & $91(20.7 \%)$ \\
\hline Technician & $28(6.4 \%)$ \\
\hline Therapist & $49(11.1 \%)$ \\
\hline \multicolumn{2}{|l|}{ Psychosocial Measures } \\
\hline Health Uncertainty (0-4) & $1.5(0.9) /$ \\
\hline "Sometimes" or more (dichotomized) & $231(52.5 \%)$ \\
\hline Anxiety (GAD-2; 0-6) & $2.5(1.8)$ \\
\hline Depression (PHQ-2; 0-6) & $1.6(1.5)$ \\
\hline Loneliness (UCLA; 2-8) & $3.3(1.5)$ \\
\hline Self-compassion (SCS; 1-5) & $3.4(1.1)$ \\
\hline Coping Confidence (MOCS-A; 2-10) & $6.8(1.4)$ \\
\hline
\end{tabular}

Note. ${ }^{*}$ Participants could select more than one option for race; ${ }^{* *} \mathrm{~N}=427$ for number of people in household.

\section{RESUlts}

A total of 440 healthcare workers completed the survey. As summarized in Table 1, the sample consisted of primarily 
white, middle-aged women. The participants were on average 44.5 years of age and predominantly identified as female (88.9\%), white (84.5\%), and non-Hispanic (90.7\%). Participants tended to live in a household with at least one other person (average $=2.8$ ). The majority of participants were nurses, medical assistants, or advanced practitioners (37.3\%), followed by those in a research or administrative role (20.7\%) and physicians $(17.7 \%)$.

Table 2. Health uncertainty among healthcare workers $(\mathrm{N}=440)$

\begin{tabular}{|c|c|c|c|}
\hline & $\begin{array}{l}\text { Health Uncertainty } \\
\text { M (SD) }\end{array}$ & $\begin{array}{l}\text { Association with Health } \\
\text { Uncertainty }\end{array}$ & $p$ \\
\hline Age & & $r=-0.05$ & .26 \\
\hline Female Gender & & $F(1,438)=0.30$ & .82 \\
\hline Race* $^{*}$ & & $F(4,435)=0.55$ & .70 \\
\hline American Indian/Alaska Native & $1.29(1.1)$ & & \\
\hline White & $1.54(.92)$ & & \\
\hline Black & $1.75(1.1)$ & & \\
\hline Asian & $1.42(.72)$ & & \\
\hline Ethnicity & & $F(1,438)=0.81$ & .37 \\
\hline Hispanic & $1.40(.93)$ & & \\
\hline Non-Hispanic & $1.56(.91)$ & & \\
\hline Number of People in Household & & $r=-0.09$ & .08 \\
\hline Job/ Clinical Role & & $F(6,433)=3.88$ & .001 \\
\hline Mental Health and Spiritual Counselor & $1.09(.79)$ & & \\
\hline Nurse, Medical Assistant, Advanced Practitioner & $1.54(.95)$ & & \\
\hline Pharmacist & $2.00(.82)$ & & \\
\hline Physician & $1.27(.80)$ & & \\
\hline Research/Administrative & $1.65(.92)$ & & \\
\hline Technician & $1.89(1.1)$ & & \\
\hline Therapist & $1.76(.72)$ & & \\
\hline \multicolumn{4}{|l|}{ Psychosocial Measures } \\
\hline Anxiety (GAD-2; 0-6) & & $r=0.41$ & $<.001$ \\
\hline Depression (PHQ-2; 0-6) & & $r=0.30$ & $<.001$ \\
\hline Loneliness (UCLA; 2-8) & & $r=0.24$ & $<.001$ \\
\hline Self-Compassion (SCS; 1-5) & & $r=0.11$ & .02 \\
\hline Coping Confidence (MOCS-A; 2-10) & & $r=-0.29$ & $<.001$ \\
\hline
\end{tabular}

Note. Associations with Health Uncertainty were computed using Pearson's $r$ (continuous variables) or ANOVA (categorical variables)

For Aim 1, the health uncertainty continuous score among the entire sample was $1.5(S D=0.91)$. When health uncertainty responses were examined categorically, health uncertainty was endorsed as present "sometimes" or more by just over half of the sample $(52.5 \%)$.

For Aim 2, there were significant associations of health uncertainty with sociodemographic, job role, and psychosocial variables (see Table 2). Health uncertainty scores were not significantly correlated with age $(r=-0.05, p=.26)$, gender $[\mathrm{F}(1,438)=0.30, p=.82]$, race $[\mathrm{F}(4,435)=.547, p=.70]$, ethnicity $[\mathrm{F}(1,438)=0.81, p=.37]$, or number of people in the household ( $r=-0.09, p=.08$ ). However, the relationship between job role and health uncertainty was statistically significant $[\mathrm{F}(6,433)=3.88, p=.001]$. Post-hoc analyses revealed significant differences were between technicians and physicians $(p=.036)$, as well as between technicians and mental health and spiritual counselors $(p=.032$; see Figure 
1). Technicians' health uncertainty levels were significantly greater than the physicians and counselors. Comparisons between pharmacists and other job roles were non-significant. All other subgroup comparisons by job role were not significant $(p>.05)$.

All psychosocial measures were significantly correlated with health uncertainty. The strongest associations were with anxiety $(r=0.41, p<.001)$ and depression $(r=0.30, p<$ $.001)$. Higher health uncertainty was also associated with more loneliness $(r=0.24, p<.001)$, yet slightly higher selfcompassion $(r=0.11, p=.02)$. In terms of coping, higher health uncertainty levels were significantly related to lower confidence in one's own ability to cope with stressors $(r=$ $-0.29, p<.001)$.

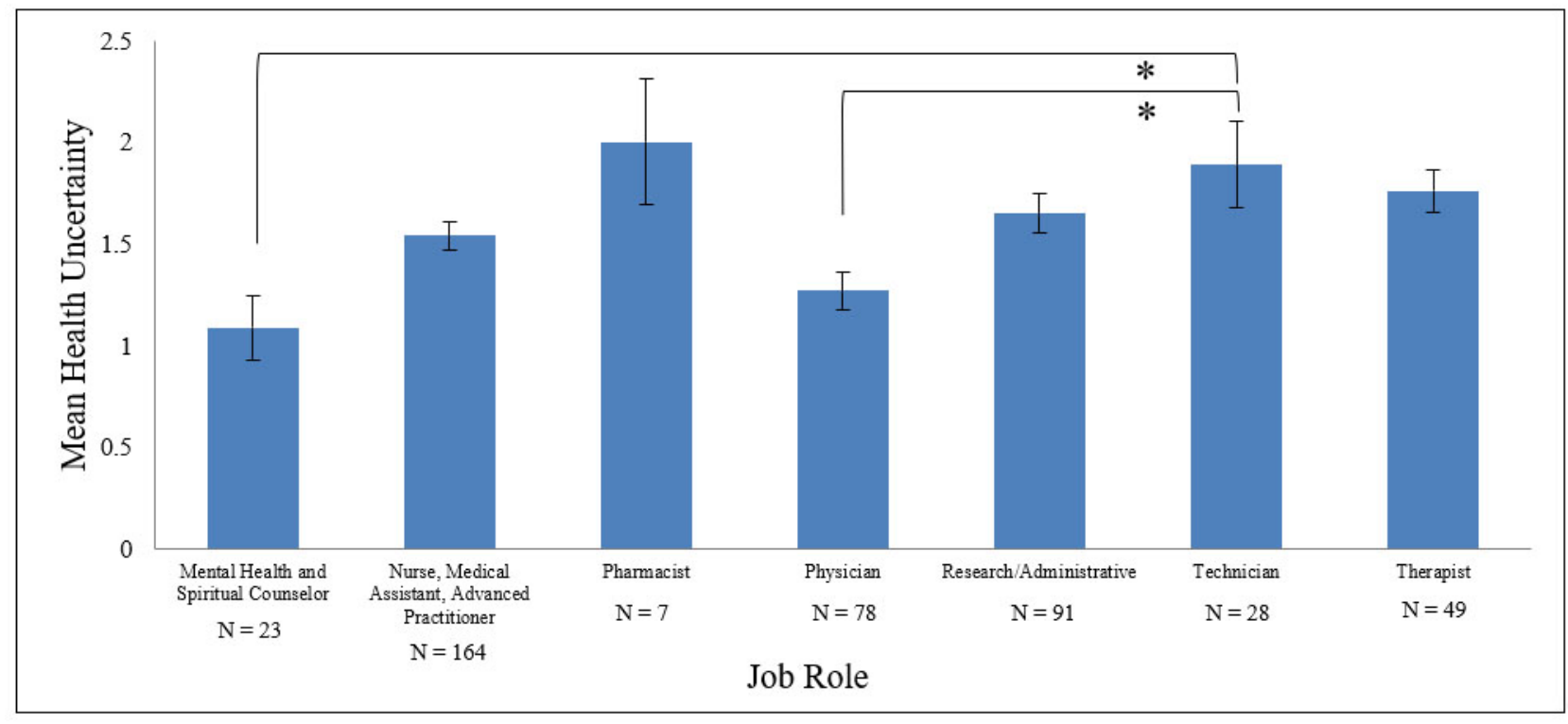

Figure 1. Mean Health Uncertainty by Job Role.

Mean scores shown for Health Uncertainty item (range 0-4). Post-hoc comparisons were computed using Tukey's HSD, with Bonferroni correction applied to adjust for multiple comparisons. Statistically significant differences are denoted by ${ }^{*}(p<.05)$

\section{Discussion}

This report is among the first to evaluate health uncertainty among healthcare workers during the initial wave of the COVID-19 pandemic in the United States. Over half of healthcare workers in this survey reported feeling uncertain about their health between "sometimes" and "all the time" during the initial wave of the COVID-19 pandemic. Higher health uncertainty was significantly associated with higher levels of anxiety, depression, and loneliness, as well as lower coping confidence. Collectively, these findings identify health-related uncertainty as a prevalent concern among healthcare workers during the COVID-19 pandemic, shed light on the subgroups of healthcare workers who may be most at risk of elevated health uncertainty, and suggest that health uncertainty is related to emotional wellbeing among healthcare providers.

Exploratory analyses examining differences by job role revealed several important, yet preliminary findings. Higher health uncertainty was more common among technicians, as compared with physicians and mental health and spiri- tual counselors. There may be relatively fewer resources available to non-clinician healthcare workers, whose psychological needs may be overshadowed by efforts to support frontline clinicians during the COVID-19 pandemic. Additionally, depending on the nature of their work, technicians may come into close contact with patients more frequently than physicians and/or spiritual counselors. These contacts may require tasks that increase technicians' risk of being exposed to COVID-19, including sanitizing patient rooms, directly administering medication, or helping patients with their personal hygiene, which could result in more health uncertainty. Pharmacists also reported high levels of health uncertainty, although comparisons with other groups were insignificant, potentially due to the small number of pharmacists $(n=7)$. This study did not stratify recruitment by job role, and, given the relatively high median uncertainty scores among pharmacists, it would have been helpful to have a larger cohort of this subgroup to more adequately power our post-hoc, between-group comparisons. Future work is needed to explore levels of health uncertainty among adequately powered sample of diverse healthcare providers, 
including technicians and pharmacists. Qualitative methods could also be used to interview healthcare workers in these roles about their perceived health risks and vulnerabilities, as well as their preferences for learning techniques to manage uncertainty.

The psychological impacts of the COVID-19 pandemic are substantial and sustained, ${ }^{[1,3,4]}$ and findings from this study indicate that healthcare workers experiencing higher health uncertainty may also be experiencing higher emotional distress. The significant associations between health uncertainty and distressing emotions, including symptoms of anxiety and depression, illustrate this link, while the negative correlation between health uncertainty and coping confidence points towards coping skills training as a critical component of psychological interventions for this population. Although we could not explicate the directionality of these associations, our findings support CBT models of health anxiety ${ }^{[16,18]}$ that indicate relationships between uncertainty, anxiety, and depression (e.g., via ruminating about the future or past) and tolerance of uncertainty as a key coping resource. The small, positive correlation between health uncertainty and self-compassion was unexpected. It is possible these constructs share similar attributes (e.g., focus on the self) that could be explicated in future studies. Longitudinal research is needed to establish whether health uncertainty is a risk factor for poorer emotional wellbeing, as well as how healthcare workers' health uncertainties may change over the course of the COVID-19 pandemic.

Healthcare workers want their organizations to understand and respond to their psychological needs during the COVID19 pandemic, including offering psychological support and tools. ${ }^{[24]}$ Evidence-based programs that promote resiliency to chronic stressors, such as one we have applied in our hospital system (Stress Management and Resiliency Training - Relaxation Response Resiliency Program; SMART-3RP), incorporate skills for tolerating uncertainty and managing chronic stress, including activating the relaxation response, practicing mindfulness, and utilizing cognitive behavioral techniques and positive psychology practices. ${ }^{[25,26]}$ Recently, the SMART-3RP was delivered to frontline COVID-19 healthcare workers and resulted in improvements in stress coping, resiliency, emotionally balanced thoughts, emotional distress, and mindfulness. ${ }^{[27]}$ Considering social distancing demands of the COVID-19 pandemic, one approach could be offering remotely-delivered (e.g., virtual) interventions with options for asynchronous content to accommodate variable shift schedules among healthcare workers occupying different clinical roles. ${ }^{[25]}$ If health uncertainty is found to impact psychological outcomes, interventions like the SMART-3RP may provide benefit by promoting tolerance of uncertainty and thereby improved psychological functioning.

This study had several strengths and limitations. Our findings are strengthened by a robust sample size of over 400 healthcare workers. Furthermore, the timing of data collection, during the initial surge of the pandemic, translates into critical insights into how to assist healthcare workers during current and future surges. However, the cross-sectional data prevents conclusions about causality between the variables. Selection bias is also a potential limitation, as all participants had signed up for an online resiliency intervention. Furthermore, we did not measure other factors related to health uncertainty, including behavioral outcomes (e.g., sleep) and proxies for COVID-19 spread near one's home (e.g., zip code). The small proportion of Black participants, a subpopulation with increased risk of serious COVID-19 symptoms, is also a significant limitation. Additionally, the job role categories were limited in scope and precluded our ability to draw comparisons between certain job roles (e.g., registered nurses vs. nurse practitioners, pharmacists who work on wards assisting medical teams vs. other settings). Finally, the adaptation of the fear of cancer recurrence measure into a health uncertainty measure could be a potential limitation, as well as the use of only selected items from validated measures, which was needed to reduce survey burden in this sample of very busy healthcare workers.

This is one of the first reports of healthcare workers' uncertainties about their health during the initial surge of COVID19 pandemic in the United States, shedding light on the need for interventions offering healthcare workers coping skills for managing health uncertainty, particularly among hospital staff whose psychosocial needs may be overlooked.

\section{ACKNOWLEDGEMENTS}

We would like to thank all the clinicians and MGH Psychiatry leadership for volunteering their time and support at the onset of the pandemic to support the needs of healthcare workers. We would like to thank the MGH Benson-Henry Institute for Mind Body Medicine, Home Base, and the Mongan Institute for provision of support for this study.

\section{FUNDING}

The following authors report financial support for the research and authorship of this article from the National Center for Complementary and Integrative Health at the National Institutes of Health (DH, K23AT010157; CML, K23AT009715; GKP, K07CA211955). LS reports financial support for the research and authorship of this article from Dauten Family as part of the Dauten Family Center for Bipolar Treatment Innovation Philanthropic Support. 


\section{CONFlicts of InTEREST Disclosure}

The authors declare they have no conflicts of interest.

\section{REFERENCES}

[1] Morgantini LA, Naha U, Wang H, et al. Factors contributing to healthcare professional burnout during the COVID-19 pandemic: A rapid turnaround global survey. PLOS ONE. 2020 Sep 3; 15(9): e0238217. PMid: 32881887. https://doi.org/10.1371/jour nal.pone. 0238217

[2] Sasangohar F, Jones SL, Masud FN, et al. Provider Burnout and Fatigue During the COVID-19 Pandemic: Lessons Learned From a High-Volume Intensive Care Unit. Anesth Analg [Internet]. 2020 Apr 20 [cited 2020 Dec 13]. Available from: https://www.ncbi .nlm.nih.gov/pmc/articles/PMC7173087/

[3] Rubin EJ, Harrington DP, Hogan JW, et al. The Urgency of Care during the Covid-19 Pandemic - Learning as We Go. N Engl J Med. 2020 Jun 18; 382(25): 2461-2. PMid: 32379956. https: //doi.org/10.1056/NEJMe2015903

[4] Adams JG, Walls RM. Supporting the Health Care Workforce During the COVID-19 Global Epidemic. JAMA. 2020 Apr 21; 323(15): 1439. PMid: 32163102. https://doi.org/10.1001/jama. 202 0.3972

[5] Wu P, Fang Y, Guan Z, et al. The psychological impact of the SARS epidemic on hospital employees in China: exposure, risk perception, and altruistic acceptance of risk. Can J Psychiatry Rev Can Psychiatr. 2009 May; 54(5): 302-11. PMid: 19497162. https://doi.org/10.1177/070674370905400504

[6] Shechter A, Diaz F, Moise N, et al. Psychological distress, coping behaviors, and preferences for support among New York healthcare workers during the COVID-19 pandemic. Gen Hosp Psychiatry. 2020; 66: 1-8. PMid: 32590254. https://doi.org/10.1016/j.genh osppsych.2020.06.007

[7] Lally J, Cantillon P. Uncertainty and ambiguity and their association with psychological distress in medical students. Acad Psychiatry. 2014 Jun; 38(3): 339-44. PMid: 24718768. https ://doi .org/10 .1007/s40596-014-0100-4

[8] Holm KE, CO D, Patterson JM, et al. The Impact of Uncertainty Associated with a Child's Chronic Health Condition on Parents' Health. Fam Syst Health J Collab Fam Healthc. 2008 Sep 1; 26(3): 282-95. PMid: 20490375. https://doi.org/10.1037/a0012912

[9] Verduzco-Aguirre HC, Babu D, Mohile SG, et al. Associations of Uncertainty With Psychological Health and Quality of Life in Older Adults With Advanced Cancer. J Pain Symptom Manage. $2021 \mathrm{Feb}$ 1; 61(2): 369-376.e1. PMid: 32822750. https://doi.org/10.1 016/j.jpainsymman $\cdot 2020 \cdot 08.012$

[10] Pappa S, Ntella V, Giannakas T, et al. Prevalence of depression, anxiety, and insomnia among healthcare workers during the COVID19 pandemic: A systematic review and meta-analysis. Brain Behav Immun. 2020 Aug; 88: 901-7. PMid: 32437915. https: //doi.org/10.1016/j.bbi.2020.05.026

[11] Galehdar N, Kamran A, Toulabi T, et al. Exploring nurses' experiences of psychological distress during care of patients with COVID19: a qualitative study. BMC Psychiatry. 2020 Oct 6; 20(1): 489. PMid: 33023535. https://doi.org/10.1186/s12888-020-0 2898-1

[12] Karampelias V, Karonis D, Psaroudi V. The psycho-emotional impact of COVID-19 on surgical staff working in emergency departments. Eur J Trauma Emerg Surg Off Publ Eur Trauma Soc. 2020 Aug;
46(4): 747-9. PMid: 32494836. https://doi.org/10.1007/s0 0068-020-01411-3

[13] Sterling MR, Tseng E, Poon A, et al. Experiences of Home Health Care Workers in New York City During the Coronavirus Disease 2019 Pandemic: A Qualitative Analysis. JAMA Intern Med. 2020 Aug 4. PMid: 32749450 . https ://doi .org/10.1001/jamainte rnmed.2020.3930

[14] Peters A, McEwen BS, Friston K. Uncertainty and stress: Why it causes diseases and how it is mastered by the brain. Prog Neurobiol 2017 Sep 1; 156: 164-88. PMid: 28576664. https ://doi.org/10 $.1016 / j$. pneurobio. 2017.05.004

[15] Lebel C, MacKinnon A, Bagshawe M, et al. Elevated depression and anxiety among pregnant individuals during the COVID-19 pandemic [Internet]. PsyArXiv; 2020 Apr [cited 2020 Dec 13]. Available from: https://psyarxiv. com/gdhkt/https://doi.org/ 10.31234/osf.io/gdhkt

[16] Salkovskis PM, Warwick HMC. Meaning, misinterpretations, and medicine: A cognitive-behavioral approach to understanding health anxiety and hypochondriasis. In: Hypochondriasis: Modern perspectives on an ancient malady. New York, NY, US: Oxford University Press; 2001. 202-22 p.

[17] McEwen BS. Stress, adaptation, and disease. Allostasis and allostatic load. Ann N Y Acad Sci. 1998 May 1; 840: 33-44. PMid: 9629234 https://doi.org/10.1111/j.1749-6632.1998.tb09546.x

[18] Luberto CM, Hall DL, Chad-Friedman E, et al. Theoretical Rationale and Case Illustration of Mindfulness-Based Cognitive Therapy for Fear of Cancer Recurrence. J Clin Psychol Med Settings. 2019 Dec; 26(4): 449-60. PMid: 30756278. https://doi .org/10.1007/s1 0880-019-09610-w

[19] Rogers SN, Cross B, Talwar C, et al. A single-item screening question for fear of recurrence in head and neck cancer. Eur Arch Oto-RhinoLaryngol Off J Eur Fed Oto-Rhino-Laryngol Soc EUFOS Affil Ger Soc Oto-Rhino-Laryngol - Head Neck Surg. 2016 May; 273(5): 123542. PMid: 25749490. https ://doi.org/10.1007/s00405-015 $-3585-\mathrm{x}$

[20] Kroenke K, Spitzer RL, Williams JBW, et al. An ultra-brief screening scale for anxiety and depression: the PHQ-4. Psychosomatics. 2009 Dec; 50(6): 613-21. https ://doi .org/10.1016/S0033-3 182(09) 70864-3

[21] Russell D, Peplau LA, Ferguson ML. Developing a measure of loneliness. J Pers Assess. 1978 Jun; 42(3): 290-4. PMid: 660402. https://doi.org/10.1207/s15327752jpa4203_11

[22] Neff KD. The Development and Validation of a Scale to Measure Self-Compassion. Self Identity. 2003 Jul; 2(3): 223-50. https: //doi.org/10.1080/15298860309027

[23] Carver CS. Measure of Current Status. 2006. Available from: http: //local.psy.miami.edu/faculty/ccarver/sclMOCS.html

[24] Shanafelt T, Ripp J, Trockel M. Understanding and Addressing Sources of Anxiety Among Health Care Professionals During the COVID-19 Pandemic. JAMA. 2020 Jun 2; 323(21): 2133. PMid: 32259193. https://doi.org/10.1001/jama. 2020.5893

[25] Hall DL, Millstein RA, Luberto CM, et al. Responding to COVID-19 Stress: Disseminating Mind-Body Resiliency Approaches. Glob Adv Health Med. 2020 Jan 1; 9: 2164956120976554 . PMid: 33312763 https://doi.org/10.1177/2164956120976554 
[26] Park ER, Traeger L, Vranceanu AM, et al. The development of a patient-centered program based on the relaxation response: the Relaxation Response Resiliency Program (3RP). Psychosomatics. 2013 Apr; 54(2): 165-74. PMid: 23352048. https://doi.org/10.101 6/j.psym.2012.09.001
[27] Park ER, Sylvia LG, Streck JM, et al. Launching a resiliency group program to assist frontline clinicians in meeting the challenges of the COVID-19 pandemic: Results of a hospital-based systems trial. Gen Hosp Psychiatry. 2020 Nov 2. PMid: 33229013. https://doi.org/10.1016/j.genhosppsych.2020.10.005 